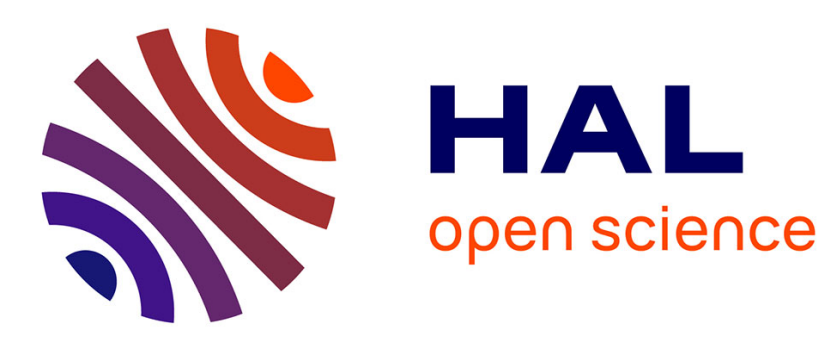

\title{
Substrats Neuronaux Impliqués dans la Perception des Actions d'Autrui. Quels Enjeux pour le Milieu Sportif? \\ Claire Calmels
}

\section{To cite this version:}

Claire Calmels. Substrats Neuronaux Impliqués dans la Perception des Actions d'Autrui. Quels Enjeux pour le Milieu Sportif?. Intellectica - La revue de l'Association pour la Recherche sur les sciences de la Cognition (ARCo), 2009, 52. hal-01575550

\section{HAL Id: hal-01575550 https: / hal-insep.archives-ouvertes.fr/hal-01575550}

Submitted on 21 Aug 2017

HAL is a multi-disciplinary open access archive for the deposit and dissemination of scientific research documents, whether they are published or not. The documents may come from teaching and research institutions in France or abroad, or from public or private research centers.
L'archive ouverte pluridisciplinaire HAL, est destinée au dépôt et à la diffusion de documents scientifiques de niveau recherche, publiés ou non, émanant des établissements d'enseignement et de recherche français ou étrangers, des laboratoires publics ou privés. 


\title{
Substrats Neuronaux Impliqués dans la Perception des Actions d'Autrui. Quels Enjeux pour le Milieu Sportif?
}

\author{
Claire Calmels*
}

\begin{abstract}
RESUME. Cette communication a tenté de mettre en évidence comment les connaissances issues du champ des neurosciences étaient en mesure d'apporter un éclairage nouveau sur les pratiques du sport de performance et plus particulièrement sur la perception des actions d'autrui. La spécificité du milieu sportif et les technologies d'investigation utilisées lors des protocoles expérimentaux ont été soulignées et ont conduit à s'interroger sur la transférabilité des connaissances issues des neurosciences au domaine sportif. Des préconisations ont été suggérées afin de faciliter ce transfert de connaissances.
\end{abstract}

Mots clés : Neurosciences, Sport de Performance, Perception

\begin{abstract}
The aim of this presentation was to show that neuroscience is of interest for the examination of perception in sports. Applying neuroscience knowledge to sport areas has been discussed and recommendations have been suggested to facilitate this application.
\end{abstract}

Keywords: Neurosciences, Elite Sport, Perception

\section{INTRODUCTION}

De manière anecdotique, il est courant de voir des spectateurs, attentifs à un match de football, réaliser des mouvements similaires à ceux qu'ils observent. Ce constat suggère le lien étroit qui existe entre la perception et l'action. L'existence de ce lien se réfère à la théorie des représentations partagées (e.g., Jeannerod, 1997; Prinz, 1997). La perception d'une action activerait dans le cerveau de l'observateur une représentation analogue à celle qu'il aurait élaborée s'il avait réalisé lui-même cette action (Grèzes, et al., 2003). Plus spécifiquement, Prinz (1997) postule dans sa théorie motrice de la perception que les évènements perçus et les actions planifiées partageraient un domaine (ou schéma) de représentation commun qui permettrait la mise en correspondance des informations sensorielles et motrices, malgré un codage spécifique et différent.

\footnotetext{
* Institut National du Sport et de l'Éducation Physique, Paris, France

Correspondant: Claire Calmels, Mission Recherche, Institut National du Sport et de l'Education

Physique, 11 Avenue du Tremblay, 75012 Paris, France.

Email: claire.calmels@insep.fr

Téléphone: (33-1) 417443 73; Fax: (33-1) 41744535
}

(C) 2009 Association pour la Recherche Cognitive. 
L'idée que les systèmes perceptif et moteur utilisent des représentations partagées n'est pas nouvelle. Cette idée est renforcée d'une part par les progrès de la technologie et l'usage de plus en plus répandu des techniques d'imagerie cérébrale (e.g., Imagerie par Résonance Magnétique Fonctionnelle, IRMf; Tomographie d'Emission de Positons, TEP) qui permettent aux chercheurs de mieux appréhender ces mécanismes. D'autre part, la découverte des neurones miroirs par l'équipe de Rizzolatti a également consolidé la compréhension du lien perception/action.

Mis en évidence au début des années 1990 dans le cortex prémoteur ventral du singe, via des enregistrements extracellulaires unitaires (e.g., Gallese et al., 1996; Rizzolatti et al., 1988), les neurones miroirs sont des neurones visuomoteurs qui présentent des caractéristiques singulières. En effet, ces neurones s'activent lorsqu'un macaque réalise une action de préhension et lorsqu'il observe cette même action réalisée par un de ses congénères ou un être humain. Par contre, ces neurones ne répondent pas lors de l'observation d'actions intransitives, d'actions mimées, d'actions réalisées avec un instrument, et d'objets seuls (voir Rizzolatti \& Craighero, 2004). L'existence d'un réseau similaire neuronal chez l'homme, mais plus complexe que celui du singe, a été mise en évidence grâce à des travaux récents d'électroencéphalographie (Calmels et al., 2008; Calmels et al., 2006; Cochin et al., 1999) et d'imagerie cérébrale (e.g., Buccino et al., 2001; Calvo-Merino et al., 2005; Calvo-Merino et al., 2006). Par exemple, Calmels et al. (2008) ont mis en évidence des patterns de synchronisation inter-cérébrale similaires en situations d'observation et d'exécution mais avec des valeurs de synchronisation plus faibles lors de l'observation. Buccino et al. (2001) ou Calvo-Merino et al. $(2005,2006)$ ont démontré, lors de l'observation d'actions, l'activation du cortex pariétal et du cortex prémoteur, aires reconnues pour appartenir au système des neurones miroirs. Pour résumer, l'appellation «neurones miroirs» s'appuie sur deux caractéristiques particulières mises en évidence chez cette population neuronale (Jacob, 2007). La première caractéristique est que ces neurones répondent, à la fois, lorsqu'un individu exécute une action et lorsqu'il observe cette même action réalisée par un de ses congénères. La seconde se traduit par leur mise en jeu, à un instant $t$, dans le cortex de deux individus, l'un réalisant une action, l'autre l'observant. Ces neurones sont localisés dans les aires frontale et pariétale et plus précisément dans la partie rostrale du lobe pariétal inférieur, dans la partie basse du gyrus précentral, et dans la partie postérieure du gyrus frontal inférieur (Rizzolatti \& Craighero, 2004).

L'objet de cet article est double. Premièrement, nous recenserons les connaissances issues de la littérature des neurosciences concernant la perception des actions d'autrui. Deuxièmement, nous centrerons notre réflexion sur l'apport de ces connaissances à la pratique sportive et à l'optimisation de la performance.

\section{Connaissances SCientifiques Concernant la Perception deS ACTIONS D'AUTRUI}

Ces dernières années, de nombreuses études dans le champ des neurosciences se sont attachées à examiner les processus sous-tendant le lien perception/action et plus spécifiquement les mécanismes de l'observation. Les caractéristiques de l'observation, la nature des instructions données avant une 
session d'observation, les caractéristiques du sujet-observateur, ou le contexte de l'observation sont quelques thématiques sur lesquelles nous allons nous attarder. Le choix de ces thématiques est motivé, d'une part, par le fait qu'elles soient perçues comme importantes pour l'optimisation de la performance par les acteurs du milieu sportif (McCullagh \& Weiss, 2001) et, d'autre part, qu'elles aient fait l'objet de recherches dans le champ des neurosciences.

\subsection{Caractéristiques de l'observation (angle d'observation)}

Kilner, Marchant, et Frith (2006) ont examiné, en utilisant la magnétoencéphalographie, l'activité du système des neurones miroirs lorsque l'angle ou la perspective d'observation était modifié. Ils se sont basés sur le postulat que l'atténuation ou encore le blocage de la puissance, détecté dans la bande de fréquence alpha et généré lors de l'observation, était un marqueur reflétant l'activité du système des neurones miroirs (Oberman et al., 2007; Pineda, 2005). Ils ont ainsi mis en évidence une diminution de puissance dans la bande de fréquence $7-12 \mathrm{~Hz}$, au niveau des sites pariétaux, lorsqu'un individu observait un acteur lui faisant face et réalisant des mouvements avec ses membres supérieurs. Ce résultat confirme ceux de la littérature (Cochin et al. 1998; Cochin et al., 1999; Babiloni et al., 2002) qui ont révélé une désynchronisation dans la bande de fréquence $8-12 \mathrm{~Hz}$ au niveau des cortices occipito-pariétal, pariétal, et sensorimoteur, lors de l'observation d'une tâche exécutée par un tiers. Kilner et al. (2006) ont également montré que lorsque l'acteur tournait le dos au sujet-observateur, aucune diminution de puissance, dans la bande de fréquence considérée, n'était relevée. En d'autres termes, les résultats de Kilner et al. (2006) suggèreraient donc l'existence d'un mécanisme qui filtrerait les informations qui parviendraient au système des neurones miroirs. Seules les informations «socialement pertinentes» $y$ auraient accès.

\subsection{Nature des instructions données avant une session d'observation}

Il a été montré qu'en fonction de la nature des instructions données avant une session d'observation, des circuits corticaux différents étaient sollicités. Grèzes et al. (1998) ont mis en évidence, avec la technique de la TEP, qu'observer sans objectif précis des actions significatives pour le sujet ou des actions dénuées de sens pour l'individu activait, en plus d'un réseau cortical identique, respectivement la voie ventrale et la voie dorsale. Par contre, l'observation d'actions significatives ou non pour l'individu mettait en jeu la voie dorsale lorsque ces actions étaient regardées dans le but de les reproduire ultérieurement. Plus précisément et toujours avec la technique de la TEP, Decety et al. (1997) ont signalé l'implication du cortex préfrontal dorsolatéral et de l'aire motrice supplémentaire lorsque des individus étaient invités à observer un mouvement dans le but de le répliquer ensuite. Le gyrus droit parahippocampique, lui, était stimulé lorsque ces mêmes individus observaient un mouvement avec l'objectif de le reconnaître ultérieurement.

Plus récemment, dans une expérience d'IRMf, Zentgraf et al. (2005) ont examiné les activations de la «SMA proper» et de la pré-SMA lors de l'observation d'une jeune fille réalisant des mouvements impliquant l'ensemble du corps (i.e., levers de bras combinés à des progressions pédestres avant et arrière). Les résultats ont révélé que:

- (i) l'activation de la «SMA proper» était plus importante lors de la condition d'observation dans le but de simuler mentalement la 
séquence perçue (OSM) que lors de la condition d'observation dont le but était d'évaluer cette séquence motrice $(\mathrm{OE})$;

- (ii) l'activation de la pré-SMA était plus prononcée lors de l'OE que lors de l'OSM.

\subsection{Caractéristiques du sujet-observateur (expertise motrice)}

Lors d'une expérience en IRMf, Buccino et al. (2004) ont constaté que les activations corticales chez l'être humain, au cours de l'observation d'actions motrices communicatives et muettes réalisées par des congénères et par des sujets d'autres espèces (i.e., singe, chien), différaient. Observer un congénère parler sans l'entendre générait chez le sujet-observateur humain une activation du pars opercularis gauche du gyrus frontal inférieur; tandis qu'observer un singe claquer les lèvres activait bilatéralement une petite partie du pars opercularis. La vue d'un chien en train d'aboyer, elle, ne produisait aucune activation statistiquement significative au sein des régions frontales. Ces résultats signifient que l'activation du système des neurones miroirs serait liée à la familiarité motrice du sujet-observateur à l'égard de l'action observée. Si cette action appartient à son répertoire moteur comportemental, le système des neurones miroirs répondra; si ce n'est pas le cas, le système des neurones miroirs restera silencieux.

Des résultats similaires ont été obtenus par Calvo-Merino et al. (2005). Des activations bilatérales plus importantes du cortex prémoteur, du cortex pariétal, et du sillon temporal supérieur (STS) ont été relevées chez des danseurs experts lors de l'observation de prestations habituellement réalisées par ces sujets alors que pour des mouvements n'appartenant pas à leur répertoire gestuel, ces activations étaient moindres (Calvo-Merino et al., 2005).

Suite au travail de Buccino et al. (2004), l'équipe de Rizzolatti a suggéré qu'une des fonctions des neurones miroirs était de participer à la compréhension d'actions (i.e., ce que fait un individu). L'engagement du système moteur, c'est-à-dire l'activation de la représentation motrice de cette action dans le cerveau de l'observateur, serait une nécessité dans ce processus de compréhension. Si le système moteur n'est pas impliqué, percevoir une action se résumera à une simple description de ses aspects visibles. Les informations (i) sur les composantes intrinsèques de cette action, (ii) sur les moyens de la réaliser, et (iii) sur ses relations avec des actions présentant des patterns communs ne seront pas disponibles (Rizzolatti, 2005). Très récemment, Rizzolatti et Sinigaglia (2008) ont précisé que deux types de compréhension pouvaient être distingués selon que le système des neurones miroirs était activé ou non. Si ce système n'est pas mis en jeu, la compréhension d'une action reposera plus sur des processus basés sur des informations visuelles. Si le système des neurones miroirs est sollicité, cette compréhension impliquera plus des processus de nature visuo-motrice.

\subsection{Contexte de l'observation}

Iacoboni, Molnar-Szakacs, Gallese, Buccino, Mazziotta, et Rizzolatti (2005) ont souligné le rôle important du contexte au sein duquel se déroule une action. Ils ont avancé qu'observer un acte de préhension dans un contexte bien défini permettait aux individus d'attribuer une intention à cet acte (i.e., pourquoi un individu réalise une action particulière). Dans une expérience d'IRMf, ils ont soumis des individus à trois conditions d'observation 
différentes. Lors de la première condition (condition contexte), les sujets observaient un certain nombre d'objets disposés sur une table et illustrant, soit le moment précédant le petit déjeuner (objets positionnés de façon ordonnée), soit le moment succédant au petit déjeuner (objets positionnés de façon désordonnée). Dans la seconde condition (condition action), les sujets regardaient la main d'une personne saisir une tasse qui était posée sur une table vide. Dans la dernière condition (condition intention), l'acte de saisie était enchâssé dans un contexte. Les sujets voyaient la main d'une personne saisir une tasse posée au milieu d'autres objets positionnés de façon ordonnée (contexte précédant le petit déjeuner) ou désordonnée (contexte succédant au petit déjeuner). Cette condition intention était supposée suggérer au sujet observateur l'intention du sujet observé c'est-à-dire soit prendre la tasse pour boire soit prendre la tasse pour la déplacer. Les résultats ont montré que le cortex frontal inférieur, où est localisé une partie des neurones miroirs, était plus fortement activé:

- (i) lors de la condition intention que lors de la condition action et de la condition contexte;

- (ii) lors de la condition intention dans le contexte précédant le petit déjeuner que lors de la condition intention dans le contexte succédant au petit déjeuner. Les résultats ont également révélé que les individus étaient parfaitement capables de distinguer chez le sujet observé son intention de boire de son intention de déplacer la tasse.

Ces résultats conduisent aux interrogations suivantes :

- 1- Comment expliquer qu'un même acte moteur (i.e., acte de saisie de la tasse) puisse occasionner une activation du système des neurones miroirs différente selon le contexte dans lequel l'individu réalise l'action?

- 2- Cela signifie-t-il que les neurones miroirs possèdent la faculté de participer à la compréhension des intentions d'autrui?

Pour tenter d'apporter des éléments de réponse à ces interrogations, Iacoboni et al. (2005) proposent un nouveau modèle: le modèle des chaînes de neurones miroirs logiquement reliés. Ils avancent que la représentation d'une intention serait basée sur l'activation d'une chaîne neuronale composée, d'une part, de neurones miroirs codant l'acte moteur observé et, d'autre part, de neurones miroirs logiquement reliés qui, eux, coderaient les actes moteurs qui seraient les plus susceptibles de se produire suite à l'observation de l'acte moteur initial, et ceci dans un contexte spécifique (Iacoboni et al., 2005). Comprendre une intention résulterait donc de l'observation de l'action mais également d'une analyse du contexte et de l'utilisation d'inférences.

\section{APPORT DES CONNAISSANCES ISSUES DE LA LITTERATURE DES NeUroscienCES AUX ACTEURS DU MILIEU SPORTIF}

Dans cette partie, trois points seront abordés. Premièrement, nous montrerons la difficulté de transférer les connaissances issues du champ des neurosciences au milieu sportif et nous en expliquerons les raisons. Deuxièmement, nous préciserons l'intérêt pour les entraîneurs, les éducateurs, ou les sportifs, de connaître les mécanismes qui sous-tendent le processus de l'observation. Dans un dernier temps, nous suggèrerons quelques 
préconisations susceptibles de faciliter le transfert des connaissances issues du champ des neurosciences au domaine sportif.

Premièrement, bien que de nombreuses avancées dans la connaissance du fonctionnement cérébral aient été réalisées lors de la dernière décennie, les applications directes en termes d'optimisation de la performance sportive sont loin d'être évidentes. Les raisons principales sont la spécificité du mouvement sportif (i.e., complexité et contextualisation) et les technologies d'investigation utilisées lors des protocoles expérimentaux.

Le mouvement sportif est complexe. La majorité des études d'imagerie cérébrale en IRM ou en PET s'est attachée à examiner, lors de sessions d'observation, principalement des tâches de saisie d'objets ou de mouvements digitaux séquentiels (e.g., Grafton et al.,1996, PET; Iacoboni et al., 2005, fMRI). Quelques rares études (6 sur 33, calcul réalisé par Turella et al., 2008) ont utilisé des tâches englobant l'ensemble du corps. Ces tâches étaient extrêmement simples et bien éloignées des réalités du milieu sportif et surtout de celles de la haute performance : des levers de bras associés à des progressions pédestres basiques (Zentgraf et al., 2005) ou des tâches de saisie où la partie inférieure du corps était dissimulée (Tai et al., 2004). Le sport de performance ne se résume pas aux tâches décrites ci-dessus. Il offre de multiples illustrations de mouvements, de tâches compliquées et complexes. Par exemple, dans des disciplines, qualifiées de morphocinèses, telles que la danse, la gymnastique, le trampoline, la natation synchronisée, la patinage, ou le plongeon, les mouvements sont extrêmement compliqués, complexes, et précis. Ils englobent l'ensemble du corps et sont réalisés autour de plusieurs axes (transversal, longitudinal, et sagittal). Dans ces disciplines de production de formes, l'incertitude environnementale est quasiment inexistante. A l'opposé, certaines disciplines (e.g., sports collectifs, sports duels) nécessitent une adaptation des sportifs à un environnement instable. Les sports collectifs en sont un bon exemple. Le joueur doit analyser, décrypter le comportement de ses adversaires mais également celui de ses partenaires. Il doit être capable de se coordonner avec ses partenaires pour mettre en place des actions afin de mettre l'équipe opposée en difficulté. Il doit également savoir déjouer les stratégies, les feintes de ses adversaires, les anticiper, et prendre des décisions en conséquence.

Le mouvement sportif est contextualisé. A notre connaissance, seule l'étude de Iacoboni et al. (2005) a examiné, chez l'être humain, les mécanismes neuronaux impliqués lors de l'observation d'une action enchâssée dans un contexte. La scène était composée d'objets (tasse, assiette avec des gâteaux, théière, pot de confiture) placés sur une table et traduisait soit un contexte précédant le petit déjeuner, soit un contexte succédant au petit déjeuner. Le contexte était inféré à partir de la disposition des objets sur la table et suggérait à l'observateur l'intention du sujet observé c'est-à-dire soit prendre une tasse pour boire, soit prendre une tasse pour la déplacer. Dans le protocole de Iacoboni et al. (2005), le contexte peut être qualifié de simple car il se rapporte à des objets. Il est immuable et figé au cours du temps, il reste dans le même état. Dans le domaine sportif, de tels contextes n'existent pas. Les sportifs évoluent avec leur propre histoire dans des environnements humain, matériel et institutionnel qui sont en constante évolution et qui donnent du sens aux actions entreprises et accomplies par le sportif. L'athlète est donc confronté à un environnement complexe au sein duquel il devra s'adapter. Si nous reprenons l'exemple des sports collectifs, lorsqu'un joueur interagit avec un 
équipier ou un adversaire, ce joueur doit être capable de comprendre son ou ses intention(s). Pour cela, il devra «prendre en compte le contexte, faire des hypothèses sur les états mentaux de l'autre, ..... se demander ce que l'autre veut lui faire faire et donc s'interroger sur la perception que l'autre a de lui» (Troubé, 2007, p.24). Plus le contexte au sein duquel se déroule une action est complexe, plus comprendre l'intention d'autrui sollicitera des ressources représentationnelles et computationnelles importantes.

Les technologies d'investigation utilisées lors des recherches en neurosciences (e.g., EEG, IRMf, PET) présentent un certain nombre de contraintes liées à leur environnement expérimental. Prenons l'exemple de la technique de l'IRMf. L'individu est couché sur le dos en décubitus dorsal dans un tube étroit et ouvert d'environ 3 mètres de long. Sa tête est entourée d'une structure métallique (antenne-tête) qui peut être dotée d'un petit miroir qui permettra à l'individu de percevoir les stimulations visuelles. La tête de l'individu est calée afin d'éviter la production de mouvements intempestifs. Des bouchons d'oreille sont insérés dans les conduits auditifs et un casque est placé sur les oreilles de l'individu dans le but d'atténuer le bruit important (120 $\mathrm{dB}$ à 1.5 Tesla et $140 \mathrm{~dB}$ à 3 Teslas) généré par les bobines de gradient pendant la phase d'acquisition. L'individu, ainsi positionné, voit sa liberté de mouvement considérablement réduite, et, en fait, selon le paradigme utilisé, il peut être amené à rester entre trente minutes et une heure dans l'IRM. Pendant l'acquisition des images, il peut réaliser des mouvements digitaux, répondre aux stimulations via un ou des bouton-pressoir(s), ou utiliser un joystick ou des claviers à cinq touches. Il ne peut se permettre d'exécuter des mouvements trop importants qui génèreraient des artéfacts et altèreraient les images IRMf. La réalisation de mouvements englobant l'ensemble du corps est, par conséquent, à exclure. La position de l'individu dans l'IRM, l'exiguïté de l'espace au sein du tunnel, et le bruit peuvent être une source d'inquiétude et d'anxiété. Ces contraintes inhérentes à tout protocole expérimental d'IRMf ne reflètent pas la réalité à laquelle sont confrontés les sportifs lorsqu'ils observent leur propre prestation ou la prestation de leur partenaire lors de sessions d'entrainement. Dans ces situations d'observation, ils sont debout ou assis, libres de leurs mouvements, et ne sont pas soumis à des sources sonores intenses. On peut donc légitimement se demander si les activations corticales enregistrées lors d'une séance d'IRMf, lorsqu'un individu perçoit des stimulations visuelles, sont similaires à celles produites lors de l'observation d'une situation sportive en contexte réel.

Nous conclurons ce premier point sur les propos de Blakemore et al., (2004) qui préconisent d'interpréter les résultats des études d'imagerie avec discernement, précaution, et sagesse car, par exemple, «ce qui est désigné par les termes de tromperie ou de moralité dans une expérience scientifique est souvent, bien loin, de refléter ce que représentent ces concepts dans la vie de tous les jours» (Blakemore et al., 2004).

Deuxièmement, la compréhension et la connaissance des mécanismes soustendant le processus de l'observation par les acteurs du milieu sportif nous semblent importantes car elles pourraient les amener à une réflexion sur leurs pratiques qui leur permettra de comprendre, d'analyser et d'optimiser la performance. Par exemple, il n'est pas essentiel pour l'entraîneur, le sportif de savoir que, lors de l'observation d'actions, une désynchronisation au niveau du cortex sensorimoteur dans la bande de fréquence $8-12 \mathrm{~Hz}$ survient (Muthukumaraswamy et al., 2004) ou que le cortex prémoteur, le sillon 
intrapariétal, le lobe supérieur pariétal droit, et le sillon temporal supérieur postérieur gauche sont activés (Calvo-Merino et al., 2005). Par contre, avoir connaissance du fait que l'observation d'une action active des zones motrices impliquées dans la planification et l'exécution du geste nous semble importante pour guider l'entraîneur dans sa pratique. Par exemple, l'inciter à mettre en place, de manière systématique, des sessions d'observation en complément des sessions traditionnelles d'entraînement physique pourrait se révéler bénéfique. Ceci pourrait permettre une diminution des charges d'entraînement, si importante en sport de haut niveau, et éventuellement réduire la fréquence des accidents et des blessures survenant dans le cadre de la pratique sportive. Dans le cadre de la rééducation des sportifs blessés, il pourrait être possible d'assister à une récupération plus rapide grâce à d'éventuelles restructurations cérébrales générées suite à l'exposition répétées de séquences sportives diverses. Présenter également des modèles possédant une expertise motrice voisine de celle du sportif semblerait plus judicieux au regard des travaux de Calvo-Merino et al. $(2005,2006)$. Ces travaux révèlent une activation plus importante du système moteur si le sujet observateur possède une expérience motrice de la tâche perçue.

De même, lors de sessions d'entraînement, l'athlète peut être amené à observer une action motrice soit pour la reproduire, soit pour identifier les erreurs lors de sa réalisation, ou encore pour apprécier l'esthétique de cette action (Zentgraf et al., 2005). Il ne nous semble pas essentiel que l'entraîneur connaisse, lors de ces différentes situations d'observation, la nature des activations au sein de la SMA, région impliquée dans la planification des actions motrices. Par contre, il est bon qu'il ait à l'esprit la distinction entre les processus élémentaires mis en jeu lors de ces situations. Observer une prestation pour la reproduire ultérieurement implique que le sujet-observateur réalise une transformation visuomotrice, c'est-à-dire transforme les informations visuelles collectées lors de l'observation en un ensemble de codes moteurs permettant la réalisation de la prestation (Jeannerod, Arbib, Rizzolatti, \& Sakata, 1995). Observer une prestation pour l'évaluer ou l'apprécier ne nécessitera pas ce genre de transformation puisque l'individu n'a pas à réaliser physiquement le geste observé. La connaissance de ces processus a pour objectif de sensibiliser les entraîneurs, les éducateurs à l'importance de donner des instructions, des consignes claires et précises avant l'observation d'un modèle et de préciser le but de cette observation. Observer une prestation pour la reproduire ou pour la reconnaître implique la mise en jeu de circuits neuronaux différents et le manque de précision dans les instructions prodiguées au sportif, à l'individu pourrait nuire à l'atteinte du but recherché.

La conclusion de ce deuxième point est que même si les préconisations proposées dans les deux exemples ci-dessus sont parfaitement connues des acteurs du milieu sportif, sans qu'il ne soit nécessaire de les informer des données issues des recherches en neurosciences, la connaissance des mécanismes mis en jeu dans le processus de l'observation par ces acteurs sportifs nous semble importante. Elle leur permet d'engager une réflexion sur leur pratique. De plus, comme l'ont souligné très justement Keil et al., (2000), sans une connaissance théorique des mécanismes sous-tendant tel ou tel processus, les raisons pour lesquelles telle ou telle intervention sportive échoue ou réussit ne seront jamais parfaitement comprises. 
Troisièmement, quelques préconisations seront proposées afin de permettre un transfert des connaissances issues du champ des neurosciences au domaine sportif plus aisé.

Une première suggestion serait de considérer, dans les procédures expérimentales, des tâches proches de celles réalisées par les sportifs. Quelques timides tentatives se sont attachées, très récemment, à prendre en considération la globalité du corps de l'individu dans des réalisations motrices complexes. Calvo-Merino et al. (2005, 2006), Cross, Hamilton, et Grafton (2006), en IRM, Babiloni et al., (2009), en EEG, ont examiné, lors de situations d'observation, les activités cérébrales au cours de séquences de danse classique, de capoiera, et de gymnastique rythmique. Ces tâches axées sur la reproduction de configurations corporelles sont caractérisées par des conditions environnementales stables et certaines. L'incertitude est inexistante. A l'opposé, certaines tâches sportives sont réalisées dans des environnements instables et peu prévisibles (e.g., sports collectifs, sports duels) où l'incertitude règne. La performance du sportif dépendra de sa capacité à s'adapter au contexte qui est en constante évolution. L'examen des bases neuronales impliquées lors de l'observation de ce second type de tâche, à la connaissance de l'auteur, n'a pas été réalisé et mériterait des investigations futures.

Une seconde suggestion serait d'observer des vidéos de mouvements sportifs en trois dimensions (3D). Cet environnement 3D, qui est l'environnement naturel de l'être humain, permettrait à ce dernier de gérer une plus grande masse d'informations visuelles que celles qu'il traite lors de la visualisation d'images en $2 \mathrm{D}$. Il vivrait ainsi des situations d'observation relativement proches de la réalité.

Une troisième suggestion serait de prendre plus en en considération, lors des procédures expérimentales, la singularité de l'individu avec son histoire personnelle, sa sensibilité, son vécu expérientiel, sa culture (Varela, 1997; Varela et al., 1993). C'est ce qu'a préconisé Varela, promoteur de la théorie de l'énaction, pour concevoir une nouvelle approche des relations entre l'individu et son environnement. Cette approche a permis de réhabiliter l'expérience humaine en considérant le rôle capital de l'enchevêtrement du cerveau et du corps, du cerveau et du contexte, et du cerveau et de la culture. En effet, Varela (1998) estime qu' «on ne peut pas comprendre la cognition si on l'abstrait de l'organisme inséré dans une situation particulière avec une configuration particulière c'est-à-dire dans des conditions écologiquement situées.» Pour appréhender l'expérience vécue, Varela (1996) et Varela et Shear (1999a, b) ont conseillé de recourir à des méthodes précises de description, d'approche à la première personne. L'entretien d'explicitation (Vermersch, 2003) pourrait être une technique permettant le recueil de ces expériences. Ce type d'entretien a pour but de faciliter la description d'une action effective et d'expliquer comment l'individu s'est organisé pour effectuer cette action. Les scientifiques pourraient ainsi coupler des démarches privilégiant une approche à la troisième personne (e.g., observation de données enregistrées via des techniques d'imagerie cérébrale) à une approche à la première personne (e.g., entretien). C'est ce qui a été mis en place par Calmels et al., (en révision) dans le cadre d'un projet sur l'étude des processus neurophysiologiques mobilisés suite à l'observation d'actions motrices. Ces auteurs, après l'acquisition de données EEG, ont effectué des entretiens d'explicitation afin de recueillir des données sur la nature des informations prélevées par des individus en situation d'observation et sur la manière dont celles-ci avaient été traitées. Il a été mis en 
évidence que les sujets utilisaient des stratégies de mémorisation diverses (e.g., répétitions vocales, répétitions visuelles, répétitions vocales associées à des répétitions visuelles). Ce constat a conduit les auteurs à répartir ces sujets dans des groupes différents en fonction de la stratégie de mémorisation utilisée. Le recours à cette procédure s'est justifié au regard des travaux de la littérature qui ont démontré l'activation d'aires corticales différentes lors de répétitions vocales et visuelles. Le cortex préfrontal gauche, le cortex occipital bilatéral, le cortex prémoteur, l'aire motrice supplémentaire (AMS) et le cortex pariétal postérieur gauche sont impliqués lors de répétitions vocales (Smith \& Jonides, 1998; Walter et al., 2003). A l'opposé, le cortex dorsal préfrontal droit, le cortex pariétal droit et le gyrus frontal moyen droit sont activés lors de l'utilisation de répétitions visuelles (Walter et al., 2003). Ainsi, l'utilisation d'une approche à la première personne a permis la réalisation de comparaisons homogènes, c'est-à-dire de comparaisons entre sujets employant des stratégies de rétention similaires.

Nous conclurons ce troisième point en soulignant que ces suggestions ne sont que des propositions visant à considérer l'individu-sportif agissant dans un environnement naturel et complexe ce qui devrait permettre une meilleure «exploitation» des données scientifiques.

\section{CONCLUSION}

En conclusion, cet article a tenté de mettre en évidence la contribution des neurosciences à l'étude de la perception des actions d'autrui dans le champ du sport de performance. La question de la transférabilité des connaissances issues de ce champ scientifique au domaine sportif a été posée et quelques préconisations ont été suggérées afin de faciliter cette transférabilité.

\section{BIBLIOGRAPHIE}

Babiloni, C., Babiloni, F., Carducci, F., Cincotti, F., Cocozza, G., Del Percio, C., Moretti, D.V., \& Rossini, P.M. (2002). Human cortical electroencephalography (EEG) rhythms during the observation of simple aimless movements: a highresolution EEG study. Neuroimage, 17, 559-572.

Babiloni, C., del Percio, C., Rossini, P.M., Marzano, N., Iacoboni, M., Infarinato, F., Lizio, R., Piazza, M., Pirritano, M., Berlutti, G., Cibelli, G., \& Eusebi, F. (2009). Judgment of actions in experts: a high-resolution EEG study in elite athletes. Neuroimage, 45,512-521.

Blakemore, S-J., Winston, J., \& Frith, U. (2004). Social cognitive neuroscience: where are we heading? Trends in Cognitive Sciences, 8, 216-222.

Buccino, G., Binkofski, F., Fink, G.R., Fadiga, L., Fogassi, L., Gallese, V., Seitz, R.J., Zilles, K., Rizzolatti, G., \& Freund, H.J. (2001). Action observation activates premotor and parietal areas in a somatotopic manner: an fMRI study. European Journal of Neuroscience, 13, 400-404.

Buccino, G., Lui, F., Canessa, N., Patteri, I., Lagravinese, G., Benuzzi, F., Porro, C.A., \& Rizzolatti, G. (2004). Neural Circuits involved in the recognition of actions performed by nonconspecifics: an fMRI study. Journal of Cognitive Neuroscience, 16, 114-126.

Calmels, C., Foutren, M., \& Stam, C. (in revision). Non linear EEG synchronization during a working memory task: Effects of instructions and expertise. International Journal of Psychophysiology. 
Calmels, C., Hars, M., Holmes, P., Jarry, G., \& Stam, C.J. (2008). Non-linear EEG synchronization during observation and execution of simple and complex sequential finger movements. Experimental Brain Research. DOI: $10.1007 / \mathrm{s} 00221-008-1480-\mathrm{z}$

Calmels, C., Holmes, P., Jarry, G., Hars, M., Lopez, E., Paillard, A., Stam, C.J. (2006). Variability of EEG synchronization prior to, and during observation and execution of a sequential finger movement. Human Brain Mapping, 27, 251-266.

Calvo-Merino, B., Glaser, D.E., Grèzes, J., Passingham, R.E., \& Haggard, P. (2005). Action observation and acquired motor skills: An fMRI study with expert dancers. Cerebral Cortex, 15, 1243-1249.

Calvo-Merino, B., Grèzes, J., Glaser, D.E., Passingham, R.E., \& Haggard, P. (2006). Seeing or doing? Influence of visual and motor familiarity in action observation. Current Biology, 16, 1905-1910.

Cochin, S., Bathelemy, C., Lejeune, B., Roux, S., \& Martineau, J. (1998). Perception of motion and qEEG activity in human adults. Electroencephalography and Clinical Neurophysiology, 107, 287-295.

Cochin, S., Barthelemy, C., Roux, S., \& Martineau, J. (1999). Observation and execution of movement: similarities demonstrated by quantified electroencephalography. European Journal of Neuroscience, 11, 1839-1842.

Cross, E.S., Hamilton A.F.C., \& Grafton, S.T. (2006). Building a motor simulation de novo: Observation of dance by dancers. Neuroimage, 31, 1257-1267.

Decety, J., Grèzes, J., Costes, N., Perani, D., Jeannerod, M., Procyk, E., Grassi, F., \& Fazio, F. (1997). Brain activity during observation of actions. Influence of action content and subject's strategy. Brain, 120, 1763-1777.

Gallese, V., Fadiga, L., Fogassi, L., \& Rizzolatti, G. (1996). Action recognition in the premotor cortex. Brain, 119, 593-609.

Grafton, S.T., Arbib, M.A., Fadiga, L., \& Rizzolatti, G., (1996). Localization of grasp representations in humans by positron emission tomography. 2. Observation compared with imagination. Experimental Brain Research, 112, 103-111.

Grèzes, J., Armony, J.L., Rowe, J., \& Passingham, R.E. (2003). Activations related to "mirror" and "canonical" neurones in the human brain: an fMRI study. Neuroimage, 18, 928-937.

Grèzes, J., Costes, N., \& Decety, J. (1998). Top-down effect of strategy on the perception of human biological motion: A PET investigation. Cognitive Neuropsychology, 15, 553-582.

Iacoboni, M., Molnar-Szakacs, I., Gallese, V., Buccino, G, Mazziotta, J.C., \& Rizzolatti, G. (2005). Grasping the intentions of others with one's own mirror neuron system. PLoS Biology 3, 529-535.

Jacob, P. (2007). Neurones miroirs, résonance et cognition sociale. Psychologie Française, 52, 299-314.

Jeannerod, J. (1997). The cognitive neuroscience of action. Oxford: Blackwell.

Jeannerod, J., Arbib, M.A., Rizzolatti, G., \& Sakata, H. (1995). Grasping objects: the cortica mechanisms of visuomotor transformation. Trends in Neurosciences, 18, 314-320.

Keil, D., Holmes, P., Bennett, S., Davids, K., \& Smith, N. (2000). Theory and practice in sport psychology and motor behaviour needs to be constrained by modelling of brain and behaviour. Journal of Sports Sciences, 18, 433-443.

Kilner, J.M., Marchant, J.L., \& Frith, C.D. (2006). Modulation of the mirror system by social relevance. SCAN, 1, 143-148.

McCullagh, P., \& Weiss, M.R. (2001). Modeling: Considerations for motor skill performance and psychological responses. In R.N. Singer, H.A. Hausenblas, \& 
C.M. Janelle (Eds.), Handbook of sport psychology (pp. 205-238). New York: Wiley.

Muthukumaraswamy, S.D., Johnson, B.W., \& McNair, N.A. (2004). Mu rhythm modulation during observation of an object-directed grasp. Cognitive Brain Research, 19, 195-201.

Oberman, L.M., McCleery, J.P., Ramachandran, V.S., \& Pineda, J.M. (2007). EEG evidence for mirror neuron activity during the observation of human and robot actions: Towards an analysis of the human qualities of interactive robots. Neurocomputing, 70, 2194-2203.

Pineda, J.A. (2005). The functional significance of mu rhythms: Translating "seeing" and "hearing" into "doing." Brain Research Reviews, 50, 57-68.

Prinz, W. (1997). Perception and action planning. European Journal of Cognitive Psychology, 9, 129-154.

Rizzolatti, G. (2005). The mirror neuron system and its function in humans. Anatomy and Embryology, 210, 419-421.

Rizzolatti, G., Carmada, R., Fogassi, L., Gentilucci, M., Luppino, G., \& Matelli, M (1988). Functional organization of inferior area 6 in the macaque monkey: II. Area F5 and the control of distal movements. Experimental Brain Research, 71, 491-507.

Rizzolatti, G., \& Craighero, L. (2004). The mirror-neuron system. Annual Review of Neuroscience, 27, 169-192.

Rizzolatti, G., \& Sinigaglia, C. (2008). Les neurones miroirs. Paris: Odile Jacob.

Smith, E.E., \& Jonides, J. (1998). Neuroimaging analyses of human working memory. Proceedings of the National Academy of Sciences USA, 95, 12061-12068.

Tai, Y.F., Scherfler, C., Brooks, D.J., Sawamoto, N., \& Castiello, U. (2004). The human premotor cortex is 'mirror' only for biological actions. Current Biology, 14, 117-120.

Troubé, S. (2007). Cognition motrice et cognition sociale: Neurones miroirs et mindreading. Mémoire de M2 non publié, Institut Jean Nicod, Paris, France.

Turella, L., Pierno, A.C., Tubaldi, F., \& Castiello, U. (2008). Mirror neurons in humans: consisting or confounding evidence? Brain and Language. DOI: 10.1016/j.bandl.2007.11.002

Varela, F. (1996). Neurophenomenology: A methodological remedy for the hard problem. Journal of Consciousness Studies, 3, 330-350.

Varela, F. (1997). Invitation aux sciences cognitives. Paris: Seuil.

Varela, F. (1998). Le cerveau n'est pas un ordinateur. La Recherche, 308, 109-112.

Varela, F., \& Shear, J. (1999a). The view from within. First-person approaches to the study of consciousness. Londres: Imprint Academic.

Varela, F., \& Shear, J. (1999b). First person methodologies: What, why, and how? In F. Varela \& J. Shear (Eds.), The view from within. First-person approaches to the study of consciousness (pp.1-14). Londres: Imprint Academic.

Varela, F., Thompson, E., \& Rosch, E. (1993). L'inscription corporelle de l'esprit. Paris: Seuil

Vermersch, P. (2003). L'entretien d'explicitation. Paris: Editions ESF.

Walter, H., Bretschneider, V., Grön, G., Zuzowski, B., Wunderlich, A.P., Tomczak, R., $\&$ Spitzer, M. (2003). Evidence for quantitative domain dominance for verbal and spatial working memory in frontal and parietal cortex. Cortex, 39, 897-911.

Zentgraf, K., Stark, R., Reiser, M., Künzell, S., Schienle, A., Kirsch, P., Walter, B. Vaitl, D., \& Munzert, J. (2005). Differential activation of pre-SMA and SMA proper during action observation: effects of instructions. Neuroimage, 26, 662-672. 